\title{
Germinación y sobrevivencia de especies arbóreas que crecen en suelos contaminados por hidrocarburos
}

\author{
J. G. Chan-Quijano, S. Ochoa-Gaona* \\ y I. Pérez-Hernández \\ Sistemas Silvícolas y Agroforestales \\ El Colegio de la Frontera Sur \\ M. A. Gutiérrez-Aguirre y J. Saragos-Méndez \\ Universidad de Quintana Roo \\ Unidad Cozumel
}

\section{Resumen}

Derivado de un estudio de prospección de especies arbóreas promisorias para la fitorremediación, se evaluó la germinación y sobrevivencia de siete especies arbóreas, las cuales fueron seleccionadas con base en su capacidad para crecer sobre suelos contaminados y en sus características de usos múltiples que puedan ser aprovechadas por los productores. Las pruebas de germinación se realizaron en un vivero establecido en las instalaciones de El Colegio de la Frontera Sur, con condiciones de humedad controlada y bajo sombra al $60 \%$. De acuerdo con las formas y características morfológicas de la testa de las semillas, se utilizaron tratamientos pregerminativos como escarificación mecánica, remojo en agua durante 24 h, inmersión en agua hirviendo, remojo en ácido sulfúrico y sin tratamiento o control. Se midió la capacidad de germinación y sobrevivencia de plántulas. Se encontró que Swietenia macrophylla, Cedrela odorata y Tabebuia rosea presentaron porcentajes de germinación mayores a 80 \% . Las tasas más altas de sobrevivencia las obtuvieron Psidium guajava, Byrsonima crassifolia, Guazuma ulmifolia, Inga inicuil y Cedrela odorata. Los datos generados proporcionan información sobre el manejo y las técnicas de germinación de las especies utilizadas, las cuales pueden ser aplicadas para la reforestación en áreas con suelos contaminados por hidrocarburos, considerando la importancia de incluir especies nativas y productoras de madera u otros productos forestales.

\section{Palabras clave}

Plántulas, reforestación, tratamiento pregerminativo, restauración.

*Correos electrónicos: j.chanquijano@hotmail.com, sochoa@ecosur.mx, iph02@hotmail.com, margutierrez@uqroo.mx,j.saragos@hotmail.com 


\title{
Germination and survival of tree species growing in soils contaminated by hydrocarbons
}

\author{
J. G. Chan-Quijano, S. Ochoa-Gaona* \\ y I. Pérez-Hernández \\ Sistemas Silvícolas y Agroforestales \\ El Colegio de la Frontera Sur \\ M. A. Gutiérrez-Aguirre y J. Saragos-Méndez \\ Universidad de Quintana Roo \\ Unidad Cozumel
}

\begin{abstract}
Derived from a prospective study of promising tree species for phytoremediation, we assessed the germination and survival of seven tree species, which were selected based on their ability to grow on contaminated soils and its multipurpose features that can be exploited by producers. Germination tests were conducted in a nursery established on the premises of El Colegio de la Frontera Sur, controlled humidity and $60 \%$ shade. According to the forms and morphological characteristics of the seed coats were used pre-germination treatments and mechanical scarification, soaking in water for $24 \mathrm{~h}$, immersion in boiling water, soaking in sulfuric acid and no treatment or control. We measured the capacity of germination and seedling survival. We found that Swietenia macrophylla, Cedrela odorata and Tabebuia rosea had germination percentages greater than $80 \%$. Survival rates over those obtained Psidium guajava, Byrsonima crassifolia, Guazuma ulmifolia, Inga inicuil and Cedrela odorata. The data generated provide information on management techniques and germination of the species used; same can be applied for reforestation in areas with soil contaminated by hydrocarbons, considering the importance of including native species and wood production.
\end{abstract}

\section{KEY WORDS}

Seedlings, reforestation, pretreatment, restoration.

*E-mail: j.chanquijano@hotmail.com,sochoa@ecosur.mx, iph02@hotmail.com,margutierrez@ uqroo.mx, j.saragos@hotmail.com 


\section{Introducción}

Hasta hace poco, en la reforestación de áreas tropicales tanto en el nivel nacional como en el local, persistía la tendencia de utilizar principalmente especies exóticas y sólo algunas especies arbóreas nativas. Sin embargo, actualmente los programas de reforestación -en particular en el estado de Tabasco- han dado mayor importancia a las especies nativas (Valle-Doménech y Ochoa-Gaona, 2008). Por ello, probar técnicas de germinación de especies que crecen en suelos contaminados por petróleo hace a éstas en principio promisorias para la fitorremediación. En este sentido, el presente estudio contribuye al fomento de especies nativas, adaptadas al medio ambiente, que pueden hacer sinergia con otros ejemplares de flora y fauna y proporcionan productos forestales maderables y no maderables a los pobladores de la región. Flores-Cuevas (2008: 62) asegura que, debido a la magnitud del problema de contaminación por hidrocarburos, es apremiante buscar soluciones redituables a corto plazo, sin generar impactos acumulativos al área afectada, por lo tanto, probar técnicas de germinación de especies promisorias para la fitorremediación encaja adecuadamente. El objetivo de este trabajo fue evaluar la capacidad de germinación y sobrevivencia de siete especies arbóreas nativas que crecen en suelos contaminados con petróleo y que las hace potenciales para la fitorremediación de los mismos.

\section{Materiales y métodos}

Ochoa-Gaona et al. (2009) realizaron un inventario florístico en zonas contaminadas con diferentes contenidos de hidrocarburos en áreas impactadas por derrames de petróleo en años recientes y anteriores de hasta 40 años en Cárdenas y Huimanguillo, Tabasco. En el informe se recomienda experimentar la germinación y sobrevivencia de las especies arbóreas encontradas. Para este estudio, se seleccionaron siete especies en función de su utilidad local, bien como frutales, cercos vivos, forraje o madera: Byrsonima crassifolia (nance), Cedrela odorata (cedro), Guazuma ulmifolia (guácimo), Inga inicuil (jinicuil), Psidium guajava (guayaba), Swietenia macrophylla (caoba) y Tabebuia rosea (macuilis). 


\section{Zona de estudio}

Ubicado en el sureste mexicano, se encuentra el estado de Tabasco, con una extensión de $24661 \mathrm{~km}^{2}$, que representa $1.3 \%$ del territorio nacional. Colinda al norte con el Golfo de México, al este con Campeche y la República de Guatemala, al sur con Chiapas y al oeste con Veracruz (InEgi, 2001: 86; Inzunza, 2005). En Tabasco, los municipios más afectados por derrames de petróleo y por consiguiente por hidrocarburos son Huimanguillo y Cárdenas. Actualmente se conocen estudios sobre el impacto de las actividades petroleras en el estado, pero se sabe con certeza que en estos dos municipios se ubican las instalaciones más antiguas de Petróleos Mexicanos (Pemex), las cuales reportan la mayor falla de materiales por su antigüedad. El clima del estado es del tipo cálido húmedo con abundantes lluvias en verano y una precipitación promedio anual de $1141 \mathrm{~mm}$ con un mínimo de $367 \mathrm{~mm}$ y un máximo de $1915 \mathrm{~mm}$. La época del año con mayores precipitaciones se presenta de junio a noviembre, periodo en que se concentra $72 \%$ de la lluvia total anual (INEGI, 2001: 86).

\section{Recolección de semillas}

Las semillas se recolectaron en remanentes de vegetación, cercas vivas, árboles dispersos y parcelas de los municipios Centro, Huimanguillo y Nacajuca, Tabasco, según las recomendaciones de Arriaga, Cervantes y Vargas-Mena (1994: 179). Una vez recolectados los frutos, se dejaron secar bajo sombra a temperatura ambiente para que abrieran y así poder sacar las semillas con facilidad, las cuales se limpiaron y secaron (Meza-Sánchez, Ruiz-Espinosa y Navejas-Jiménez, 2009: 28).

\section{Tratamientos aplicados}

A las semillas de cada especie se les aplicaron cuatro tratamientos pregerminativos y un lote de control al que no se le aplicó tratamiento y que funcionó como testigo. Para cada tratamiento se emplearon 30 semillas de cada especie, lo que representó 150 semillas por tratamiento, evaluando un total de 1050 semillas. De acuerdo con las formas y características morfológicas de la testa de las semillas descritas por Stearn (1992: 546), y con las particularidades del embrión y los cotiledones de 
las semillas definidas por Niembro-Rocas (1988: 286), se aplicaron diferentes tratamientos pregerminativos, siendo los más utilizados: a) escarificación mecánica (T1), b) remojo en agua durante 24 h (T2), c) inmersión en agua hirviendo (T3), d) remojo en ácido sulfúrico (T4) y e) sin tratamiento o control. Los datos se monitorearon cada dos días registrando el número de semillas germinadas y la sobrevivencia de plántulas. El número de días en que se dio seguimiento fue variable para cada especie.

Esta investigación se llevó al cabo en un vivero establecido en las instalaciones de El Colegio de la Frontera Sur, Unidad Villahermosa, Tabasco, con sombra al 60 \% y con facilidades de riego (Hermosillo-González et al., 2008). Las semillas se sembraron en camas de germinación de 4 m x 1 m cada una, utilizando como base grava sobre plástico negro para evitar el crecimiento de malezas y así retener la humedad; una capa de arena de $10 \mathrm{~cm}$, para evitar la humedad excesiva, y por arriba un sustrato compuesto de suelo con cascarilla de cacao en proporción 1:1. Las semillas $\geq 1 \mathrm{~cm}$ se sembraron a una distancia de $20 \mathrm{~cm}$ entre filas y columnas; en cada punto se sembraron tres semillas. Las semillas $<1 \mathrm{~cm}$ se sembraron en bolsas negras de polietileno de $20 \mathrm{~cm}$ de largo x $10 \mathrm{~cm}$ de diámetro, con el objetivo de evitar su pérdida, como podría suceder en las camas; conforme fueron germinando, las plántulas se etiquetaron con un popote de plástico (Pérez-Hernández et al., 2011).

\section{Análisis de los datos}

A los datos de germinación y sobrevivencia obtenidos se les aplicó la prueba estadística de Kruskal-Wallis (Daniel, 1995: 875; Blanco, 2000), la cual nos ayudó a determinar las posibles diferencias significativas entre tratamientos para cada especie.

\section{Resultados}

Las semillas de las especies respondieron de manera distinta a cada uno de los tratamientos, y la prueba estadística mostró que las diferencias fueron significativas (cuadro 1). Swietenia macrophylla, Cedrela odorata y Tabebuia rosea presentan porcentajes promedio de germinación mayores a $80 \%$. Los menores valores los tuvo Guazuma ulmifolia. 
Cuadro 1. Prueba de Kruskal-Wallis de porcentaje de germinación entre distintos tratamientos, por especie

$\begin{array}{lccc}\text { Especie } & \text { N* } & \begin{array}{c}\text { Estadístico de } \\ \text { prueba (H) }\end{array} & \text { P } \\ \text { Byrsonima crassifolia (nance) } & 130 & 16.26 & 0.0027 \\ \text { Cedrela odorata (cedro) } & 135 & 47.63 & 0.0000 \\ \text { Guazuma ulmifolia (guácimo) } & 165 & 108.45 & 0.0000 \\ \text { Inga inicuil (jinicuil) } & 85 & 61.37 & 0.0000 \\ \text { Psidium guajava (guayaba) } & 130 & 85.16 & 0.0000 \\ \text { Swietenia macrophylla (caoba) } & 190 & 31.98 & 0.0000 \\ \text { Tabebuia rosea (macuilis) } & 115 & 46.026 & 0.0000\end{array}$

${ }^{*} \mathrm{~N}$ : Tamaño de la muestra.

Para Swietenia macrophylla la germinación de semillas en todos los tratamientos alcanzó de 86 a 93 \%. El mayor valor lo presentó el SmT2 a los diez días después de la siembra (cuadro 2).

Cuadro 2. Días requeridos para iniciar la germinación y porcentaje de germinación total por tratamiento para Swietenia macrophylla

\begin{tabular}{llcc} 
Tratamiento & Descripción de tratamiento & $\begin{array}{c}\text { Días de inicio } \\
\text { de germinación }\end{array}$ & $\begin{array}{c}\text { Capacidad de } \\
\text { germinación } \\
\text { (\%) }\end{array}$ \\
SmC & Control & 24 & 86 \\
SmT1 & Escarificación mecánica (EM) & 6 & 86 \\
SmT2 & $\begin{array}{l}\text { Remojo en agua durante 24 } \\
\text { h (R24) }\end{array}$ & 10 & 93 \\
SmT3 & $\begin{array}{l}\text { Escarificación mecánica y } \\
\text { remojo en agua durante 24 h } \\
\text { (EMR24) }\end{array}$ & 6 & 86 \\
SmT4 & $\begin{array}{l}\text { Remojo en agua durante } 48 \\
\text { h (R48) }\end{array}$ & 10 & 90 \\
\hline
\end{tabular}


Germinación y sobrevivencia de especies arbóreas que crecen en suelos contaminados por hidrocarburos

Cedrela odorata mostró respuesta diferencial a los tratamientos: el CoT1 con el mayor valor de germinación, mientras que el CoT4 con el menor (73\%). La germinación de esta especie comenzó entre el cuarto y el sexto día después de la siembra (cuadro 3).

Cuadro 3. Días requeridos para iniciar la germinación y porcentaje de germinación total por tratamiento para Cedrela odorata

\begin{tabular}{llcr} 
Tratamiento & \multicolumn{1}{c}{ Descripción de tratamiento } & $\begin{array}{c}\text { Días de inicio } \\
\text { de germinación }\end{array}$ & $\begin{array}{c}\text { Capacidad de } \\
\text { germinación } \\
\text { (\%) }\end{array}$ \\
CoC & Control & 6 & 83 \\
CoT1 & Remojo en agua durante 6 h (R6) & 6 & 100 \\
CoT2 & $\begin{array}{l}\text { Remojo en agua durante 12 h } \\
\text { (R12) }\end{array}$ & 4 & 96 \\
CoT3 & $\begin{array}{l}\text { Remojo en agua durante 24 h } \\
\text { (R24) }\end{array}$ & 4 & 90 \\
CoT4 & $\begin{array}{l}\text { Remojo en agua durante 34 h } \\
\text { (R34) }\end{array}$ & 4 & 73 \\
\hline
\end{tabular}

Cuadro 4. Días requeridos para iniciar la germinación y porcentaje de germinación total por tratamiento para Tabebuia rosea

\begin{tabular}{llcc} 
Tratamiento & \multicolumn{1}{c}{$\begin{array}{c}\text { Descripción de } \\
\text { tratamiento }\end{array}$} & $\begin{array}{c}\text { Días de inicio de } \\
\text { germinación }\end{array}$ & $\begin{array}{c}\text { Capacidad de } \\
\text { germinación } \\
\text { (\%) }\end{array}$ \\
$\operatorname{TrC}$ & Control & 6 & 73 \\
$\operatorname{TrT} 1$ & $\begin{array}{l}\text { Remojo en agua durante } \\
\text { 6 h (R6) }\end{array}$ & 6 & 86 \\
$\operatorname{TrT2}$ & $\begin{array}{l}\text { Remojo en agua durante } \\
12 \text { h (R12) }\end{array}$ & 6 & 93 \\
$\operatorname{TrT3}$ & $\begin{array}{l}\text { Remojo en agua durante } \\
24 \text { h (R24) } \\
\text { Remojo en agua durante }\end{array}$ & 4 & 83 \\
TrT4 & 34 h (R34) & 4 & 96 \\
& & & \\
\hline
\end{tabular}


Los mayores valores de germinación para Tabebuia rosea se observaron en $\operatorname{TrT} 2$ y TrT4 (93 y $96 \%$, respectivamente). El menor valor de germinación se obtuvo en el control $(\operatorname{TrC})$. Las semillas de esta especie empezaron a germinar entre el cuarto y el sexto día después de la siembra (cuadro 4).

Inga inicuil logró la mayor germinación en IiT1 e IiT2 con 83 y 80 \%, respectivamente, mientras que la menor se presentó en el control (IiC). Esta especie inició la germinación a los dos días después de la siembra (cuadro 5). Se encontraron diferencias significativas en el porcentaje de germinación de Inga inicuil entre tratamientos.

Cuadro 5. Días requeridos para iniciar la germinación y porcentaje de germinación total por tratamiento para Inga inicuil

\begin{tabular}{llcc} 
Tratamiento & Descripción de tratamiento & $\begin{array}{c}\text { Días de } \\
\text { inicio de } \\
\text { germinación }\end{array}$ & $\begin{array}{c}\text { Capacidad de } \\
\text { germinación } \\
(\%)\end{array}$ \\
IiC & Control & 2 & 36 \\
IiT1 & Remojo en agua durante 6 h (R6) & 2 & 83 \\
IiT2 & Remojo en agua durante 12 h (R12) & 2 & 80 \\
IiT3 & Remojo en agua durante 24 h (R24) & 2 & 53 \\
IiT4 & Remojo en agua durante 34 h (R34) & 2 & 53 \\
\hline
\end{tabular}

Para Guazuma ulmifolia los tratamientos GuT3 y GuT4 exhibieron los porcentajes más altos de germinación (83 y $90 \%$, respectivamente). Los otros tratamientos reportaron valores muy bajos. Esta especie comenzó la germinación entre el cuarto y el octavo día después de la siembra en todos los tratamientos (cuadro 6).

El mayor porcentaje de germinación para Byrsonima crassifolia se manifestó en el BcT4 (60 \%) y el menor en BcT1 (1 \%). Su germinación empezó entre el día ocho y el día 14 para todos los tratamientos (cuadro 7).

Para Psidium guajava la mayor proporción de semillas germinadas se presentó en el control PgC con 93 \%, seguida del PgT1. En el PgT3 y el PgT4 no hubo semillas germinadas (0\%). Esta especie comenzó a germinar entre el día seis y el día 12 (cuadro 8). 
Germinación y sobrevivencia de especies arbóreas que crecen en suelos contaminados por hidrocarburos

Cuadro 6. Días requeridos para iniciar la germinación y porcentaje de germinación total por tratamiento para Guazuma ulmifolia

\begin{tabular}{|c|c|c|c|}
\hline Tratamiento & Descripción de tratamiento & $\begin{array}{l}\text { Días de inicio } \\
\text { de germinación }\end{array}$ & $\begin{array}{l}\text { Capacidad de } \\
\text { germinación } \\
(\%)\end{array}$ \\
\hline $\mathrm{GuC}$ & Control & 8 & 13 \\
\hline GuT1 & $\begin{array}{l}\text { Escarificación química con ácido } \\
\text { sulfúrico por } 3 \text { min y lavado de semilla } \\
\text { (EQAS2MLS) }\end{array}$ & 8 & 13 \\
\hline GuT2 & $\begin{array}{l}\text { Remojo por } 30 \text { seg en agua caliente más } \\
\text { remojo en agua a } \mathrm{T}^{\circ} \text { ambiente por } 24 \mathrm{~h} \\
\text { y lavado de semilla (R30ACR24LS) }\end{array}$ & 4 & 6 \\
\hline GuT3 & $\begin{array}{l}\text { Remojo con agua hirviendo durante } \\
10 \text { min más remojo en agua a } \mathrm{T}^{\circ} \\
\text { ambiente por } 24 \mathrm{~h} \text { y lavado de semilla } \\
\text { (RAH10R24LS) }\end{array}$ & 4 & 83 \\
\hline GuT4 & $\begin{array}{l}\text { Remojo con agua caliente durante } \\
10 \text { min más remojo en agua a } \mathrm{T}^{\circ} \\
\text { ambiente por } 24 \mathrm{~h} \text { y lavado de semilla } \\
\text { (RAC10R24LS) }\end{array}$ & 4 & 90 \\
\hline
\end{tabular}

Cuadro 7. Días requeridos para iniciar la germinación y porcentaje de germinación total por tratamiento para Byrsonima crassifolia

\begin{tabular}{clrr} 
Tratamiento & \multicolumn{1}{c}{ Descripción de tratamiento } & $\begin{array}{c}\text { Días de inicio } \\
\text { de germinación }\end{array}$ & $\begin{array}{c}\text { Capacidad de } \\
\text { germinación } \\
(\%)\end{array}$ \\
\hline BcC & Control & 12 & 43 \\
BcT1 & Escarificación mecánica (EM) & 8 & 1 \\
BcT2 & $\begin{array}{l}\text { Escarificación química con ácido } \\
\text { sulfúrico por 2 min y lavado de semilla } \\
\text { (EQAS2MLS) }\end{array}$ & 14 & 43 \\
BcT3 & $\begin{array}{l}\text { Escarificación química con ácido } \\
\text { sulfúrico por 3 min y lavado de semilla } \\
\text { (EQAS3MLS) }\end{array}$ & 14 & \\
BcT4 & $\begin{array}{l}\text { Escarificación química con ácido } \\
\text { sulfúrico por 3 min más remojo durante } \\
\text { 24 h (EQAS3MR24) }\end{array}$ & 14 & 60 \\
\hline
\end{tabular}


Cuadro 8. Días requeridos para iniciar la germinación y porcentaje de germinación total por tratamiento para Psidium guajava

\begin{tabular}{llcc} 
Tratamiento & \multicolumn{1}{c}{ Descripción de tratamiento } & $\begin{array}{c}\text { Días de } \\
\text { inicio de } \\
\text { germinación }\end{array}$ & $\begin{array}{c}\text { Capacidad de } \\
\text { germinación } \\
(\%)\end{array}$ \\
PgC & $\begin{array}{l}\text { Control } \\
\text { PgT1 }\end{array}$ & $\begin{array}{l}\text { Remojo en agua a T ambiente } \\
\text { durante 34 h (R34) }\end{array}$ & 63 \\
PgT2 & $\begin{array}{l}\text { Remojo en agua hirviendo por } \\
\text { 30 seg (RAH30S) }\end{array}$ & 12 & 66 \\
PgT3 & $\begin{array}{l}\text { Remojo con agua caliente por } \\
\text { 5 min y lavado de semilla } \\
\text { (RAC5MLS) }\end{array}$ & 0 & 0 \\
PgT4 & $\begin{array}{l}\text { Remojo con agua caliente } \\
\text { durante 10 min más remojo en } \\
\text { agua a T }{ }^{\circ} \text { ambiente durante }\end{array}$ & 0 & 0 \\
& $\begin{array}{l}\text { 24 h y lavado de semilla } \\
\text { (RAH10R24LS) }\end{array}$ & & \\
\hline
\end{tabular}

\section{Porcentaje final de sobrevivencia de plántulas}

De acuerdo con los resultados obtenidos en la sobrevivencia de plántulas, las siete especies estudiadas presentaron diferencias significativas en función de los tratamientos aplicados (cuadro 9 y figura 1).

En general, la mayor sobrevivencia promedio de plántulas la presentaron Swietenia macrophylla y Tabebuia rosea con 90 \%, seguidas por el resto de especies con un promedio de 67 a $70 \%$. Byrsonima crassifolia mostró el mayor valor de sobrevivencia en el $\mathrm{BcC}$ con $70 \%$ (figura 1A), y Cedrela odorata en el CoC con $100 \%$ (figura 1B). Para Guazuma ulmifolia la mayor sobrevivencia se observó en GuT2 (97 \%) y la menor en GuT4 (63 \% , figura 1C); para Inga inicuil, la mayor en IiC (97\%) y la menor en IiT4 (77 \% , figura 1D); y para Tabebuia rosea, la mayor en $\operatorname{TrT} 4$ (100 \%) y la menor en $\operatorname{TrT3}(93 \%$, figura 1E). Psidium guajava exhibió la mayor sobrevivencia en PgT2 con $70 \%$, en tanto que el control y el resto de los tratamientos presentaron un promedio de sobrevivencia de $66 \%$ (figura 1F). Por último, en 
Germinación y sobrevivencia de especies arbóreas que crecen en suelos contaminados por hidrocarburos

Cuadro 9. Prueba de Kruskal-Wallis de sobrevivencia entre los distintos tratamientos, por especie

\begin{tabular}{lccc} 
Especie & N* & $\begin{array}{c}\text { Estadístico de } \\
\text { prueba }(\mathrm{H})\end{array}$ & $\mathrm{P}$ \\
Byrsonima crassifolia (nance) & 130 & 19.28 & 0.0001 \\
Cedrela odorata (cedro) & 135 & 118.32 & 0.0000 \\
Guazuma ulmifolia (guácimo) & 164 & 157.53 & 0.0000 \\
Inga inicuil (jinicuil) & 84 & 60.46 & 0.0000 \\
Psidium guajava (guayaba) & 130 & 10.54 & 0.0322 \\
Swietenia macrophylla (caoba) & 190 & 145.74 & 0.0000 \\
Tabebuia rosea (macuilis) & 114 & 79.46 & 0.0000 \\
\hline
\end{tabular}

*N: Tamaño de la muestra.

Swietenia macrophylla se advirtió 100 \% de sobrevivencia en SmT1, SmT2 y SmT3, mientras que el menor valor lo manifestó SmT4 con 93 \% (figura 1G).

\section{Discusión}

\section{Evaluación de germinación de semillas con tratamientos de remojo en agua}

Los tratamientos de remojo en agua a temperatura ambiente son considerados excelentes ya que hidratan la semilla después de la conservación y así la germinación será más efectiva. Respecto a Swietenia macrophylla, una de las técnicas más recomendables para que alcance una alta capacidad de germinación es el remojo en agua, pues se reduce el tiempo para lograr la máxima germinación (NiembroRocas y Ramírez-García, 2006: 21-28), lo cual se observó en el SmT4 (remojo en agua durante 48 h) y el SmT2 (remojo en agua durante $24 \mathrm{~h}$ ), donde se encontraron porcentajes más altos. El control (SmC), con $86 \%$ de germinación, indica que sin aplicar ningún tratamiento la especie puede germinar sin problema alguno. Guerrero y Herrera (1994) reportaron que la escarificación favorece la germinación de 

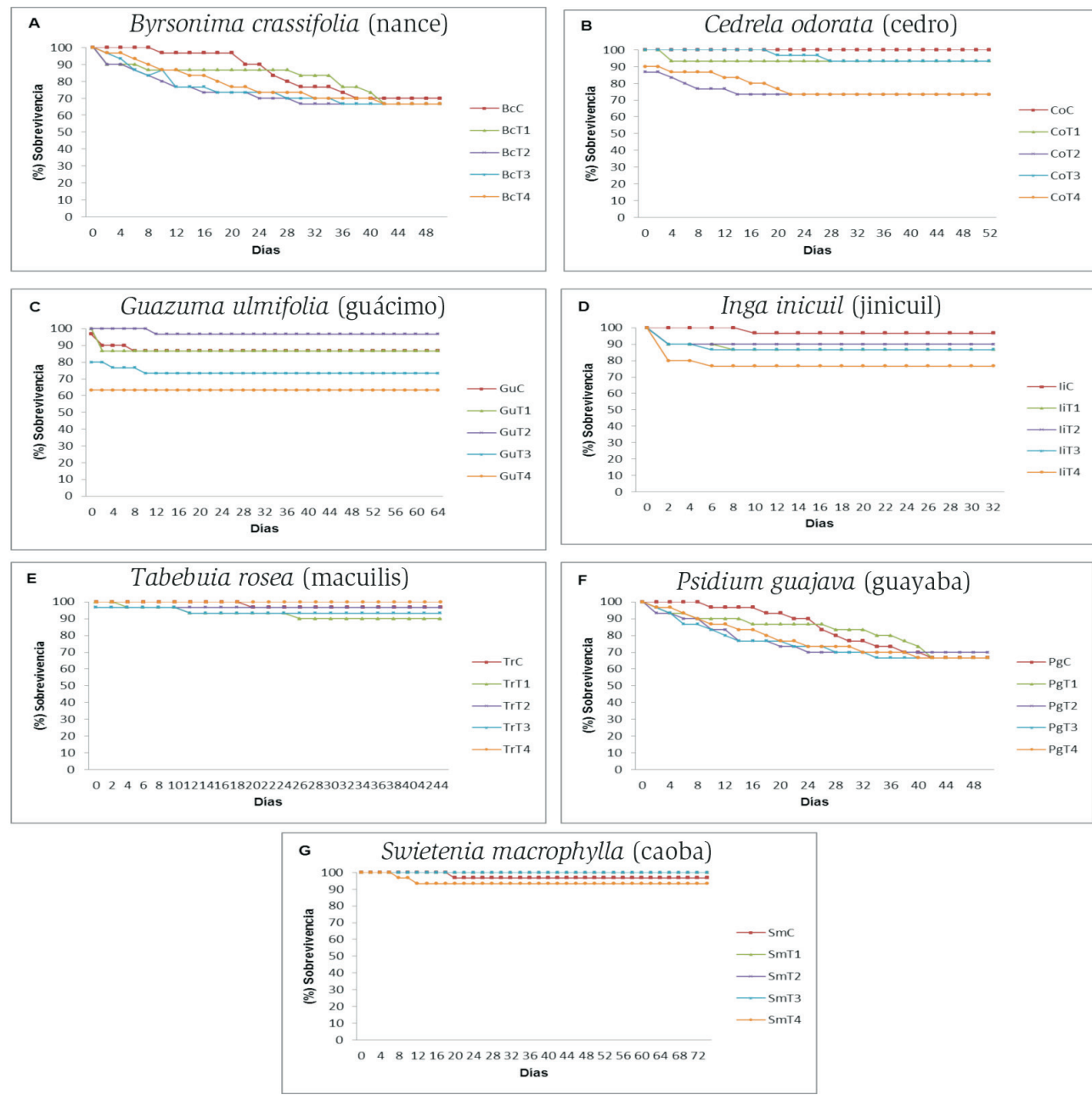

Figura 1. Tasa de sobrevivencia: Byrsonima crassifolia (A), Cedrela odorata (B), Guazuma ulmifolia (C), Inga inicuil (D), Tabebuia rosea (E), Psidium guajava (F) y Swietenia macrophylla (G) 
Cedrela odorata es una especie con potencial para la reforestación en zonas degradadas de selva; en su distribución natural no es posible encontrar árboles de gran talla y buena forma debido a la gran explotación a la que ha estado sujeta durante más de 200 años. Asimismo, Perozo-Castro et al. (2003) mencionan que las plántulas de las especies arbóreas presentan un crecimiento lento, pues encontraron que, con 56 días de germinación, las plántulas sólo alcanzaron $7.7 \mathrm{~cm}$ de crecimiento, fenómeno que especialmente no sucedió con Cedrela odorata, ya que en este estudio se obtuvo una altura final de $15.6 \mathrm{~cm}$ en 52 días (CoC); además, en esta investigación se evidenció que el tratamiento de remojo en agua durante $6 \mathrm{~h}$ (CoT1) es el más recomendable (14.2\%), aunque los otros tratamientos también son bastante aconsejables, pues alcanzaron porcentajes mayores a 70 \% (Arriaga, Cervantes y Vargas-Mena, 1994: 179).

En cuanto a Tabebuia rosea, Biota Panamá (2008) menciona que una forma de mantener la viabilidad de sus semillas es conservarlas en refrigeración, proceso que facilita su manejo en el nivel local por los campesinos y productores rurales. Con base en los resultados, la germinación con el tratamiento de remojo en agua durante 34 h (96\% de germinación) es la más conveniente. Según Salvador-Figueroa, Adriano-Anaya y Becerra-Ortiz (2005), el incremento en el tiempo de remojo favorece tanto el inicio como el porcentaje total de emergencia de plántulas.

De acuerdo con Salvador-Figueroa, Adriano-Anaya y Becerra-Ortiz (2005) y Vargas-Simón, Pire y de la O de la O (2009), Inga inicuil presenta porcentajes intermedios de germinación, como puede apreciarse en IiT1 (remojo en agua durante 6 h) e IiT2 (remojo en agua durante $12 \mathrm{~h}$ ); en ambos casos existe una conexión de la semilla con el agua y, en función de nuestros resultados, estos dos tratamientos son los recomendados ya que aumentan las probabilidades de germinación.

Para estas especies nativas, sus semillas siguen siendo el método de propagación más empleado en vivero (Ochoa-Gaona et al., 2008: 98; Quiroz-Marchant et al., 2009: 128). Por ello, es de suma importancia disponer desde un comienzo con semillas de buena calidad. De tal suerte, los tratamientos pregerminativos aplicados a estas especies cobran una gran relevancia, ya que a partir de sus resultados se podrá tener los mejores tratamientos para que las semillas germinen exitosamente, ya que cada comunidad vegetal presenta mecanismos de germinación característicos que responden al efecto de la selección natural inducida por las condiciones 
ambientales predominantes sobre la naturaleza, los viveros y la fisiología de las semillas.

\section{Evaluación de tratamientos con escarificación química, remojo en agua caliente e hirviendo}

Otros trabajos realizados con diferentes especies arbóreas han encontrado que la escarificación con ácido sulfúrico permite incrementar la proporción de semillas germinadas (Padma, Reddy y Satyanarayana, 1995). Sin embargo, en el presente experimento, para Guazuma ulmifolia los tratamientos con agua hirviendo y caliente mostraron los mejores porcentajes (GuT3 $=83 \%$ y GuT4 $=90 \%$ ), mientras que el tratamiento de escarificación química con ácido sulfúrico presentó baja cantidad de semillas germinadas (GuT1 $=13 \%$ y GuT2 $=6 \%$ ). El agua hirviendo y la caliente ayudan a eliminar el mucilago que recubre las semillas contribuyendo a que se inicie la germinación. Por el contrario, los tratamientos con ácido sulfúrico no la propiciaron, tal vez porque las semillas no soportaron el ácido y éste causó daños a los cotiledones o al embrión (Hernández-Vargas, Sánchez-Velásquez y Aragón, 2001; Hermosillo-González et al., 2008; Hernández-Gil, Rada y Silva, 2010).

La escarificación química (ácido sulfúrico) elevó la germinación de Byrsonima crassifolia, obteniendo porcentajes medios de acuerdo con Arriaga, Cervantes y Vargas-Mena (1994: 179). En este caso, el tratamiento recomendable es BcT4 (escarificación química con ácido sulfúrico por 3 min más remojo en agua durante 24 h), pues presentó el porcentaje más alto de germinación en comparación con el resto de los tratamientos, probablemente debido a que el ácido facilitó el rompimiento de la estructura externa de la semilla y el remojo en agua durante 24 h ayudó a la hidratación de las semillas provocando la germinación. El remojo en agua es un método sustitutivo que difícilmente producirá una germinación alta para esta especie; es decir, este método podría ser empleado por el pequeño agricultor, aceptando las grandes pérdidas de semillas no germinadas y la desuniformidad de las plantas, pero sería una alternativa poco eficiente; en cambio, la escarificación química con el ácido sulfúrico induce la germinación de estas semillas y proporciona porcentajes favorables (Camino, 1998: 15). No obstante, aquellos agricultores que produzcan 
esta especie corren el riesgo de perder semillas, ya que por el momento hay que probar nuevos tratamientos para mejorar la germinación.

Los tratamientos aplicados a Psidium guajava tuvieron poco efecto sobre la capacidad de germinación, posiblemente porque el tiempo de inmersión mató al embrión o porque no se logró romper la testa, que es muy dura. Castellano et al. (2006) obtuvieron datos similares. Tal vez el calor no sea la solución para esta semilla. De tal modo, se recomienda utilizar semillas sin tratamientos pregerminativos, puesto que el control (PgC) presentó un alto porcentaje de germinación.

El comportamiento de las especies dentro del vivero se debe a que las semillas permanecieron en un ambiente más controlado. Los días requeridos para el inicio de la germinación sugieren que este proceso es rápido, lo cual pudo suceder porque las semillas fueron sembradas casi de inmediato después de la recolección sin modificar su viabilidad (Arriaga, Cervantes y Vargas-Mena, 1994: 179; Pérez-Hernández et al., 2011). Empero, es posible que también las diferencias morfológicas de las semillas y la aplicación de tratamientos pregerminativos facilitaran la germinación, sobre todo para las semillas ortodoxas como Guazuma ulmifolia, Psidium guajava y Byrsonima crassifolia.

\section{Evaluación de la sobrevivencia (\%) de las siete especies arbóreas}

La sobrevivencia es una etapa crítica en las plántulas tanto en ambientes naturales como en plantaciones y programas de reforestación (Meza-Sánchez, Ruiz-Espinosa y Navejas-Jiménez, 2009: 28). Swietenia macrophylla y Tabebuia rosea muestran que el tratamiento de remojo en agua alcanza la mayor proporción de plántulas sobrevivientes para casi todas las especies; por el contrario, la escarificación química con ácido sulfúrico y el remojo en agua caliente e hirviendo exhibieron la menor sobrevivencia de la mayoría de las especies (Burbano, 1990), resultados que se observaron debido a que se pudo dañar la testa de la semilla.

Psidium guajava, Byrsonima crassifolia, Guazuma ulmifolia, Inga inicuil y Cedrela odorata presentan un promedio de sobrevivencia similar en los tratamientos, tal vez porque estos ejemplares sólo fueron sumergidos en agua en diferentes intervalos. Martínez (1986), Chaves et al. (1999), Zamora-Cornelio et al. (2010) y Pérez-Hernández et al. (2011) reportan un crecimiento medio anual en la altura y el diámetro de estas especies bajo condiciones de tratamientos y en vivero. 


\section{Conclusiones}

Cuando menos en alguno de los tratamientos aplicados la mayoría de las especies presentó valores de germinación altos ( $>=76 \%$ ), de acuerdo con el criterio de Arriaga, Cervantes y Vargas-Mena (1994: 179).

Para la mayoría de las especies, la escarificación y el remojo en agua (por tiempo variable) resultaron las técnicas con porcentajes de germinación elevados, por lo cual, al ser baratas y accesibles para viveros forestales, serían las más recomendadas. En particular, el guácimo (Guazuma ulmifolia) y el nance (Byrsonima crassifolia) requieren tratamientos más sofisticados. El macuilis (Tabebuia rosea), la caoba (Swietenia macrophylla) y el jinicuil (Inga inicuil) mostraron las mayores tasas de sobrevivencia.

\section{FuENTES CONSULTADAS}

Arriaga, M. V., V. G. Cervantes y A. Vargas-Mena (1994). Manual de reforestación con especies nativas: colecta y preservación de semillas, propagación y manejo de plantas. México: Secretaría de Desarrollo Social/Instituto Nacional de Ecología/Universidad Nacional Autónoma de México.

Biota Panamá (2008). Cultivo de árboles nativos de Panamá [en línea]. Panamá: Biota Panamá. Disponible en: http://biota.wordpress.com/2008/12/25/el-cultivo-del-roble-de-sabana-tabebuia-rosea/ [2012, 28 de julio].

Blanco, F. A. (2000). "Métodos apropiados de análisis estadísticos subsiguientes al análisis de varianza (ANDEVA)”. Agronomía Costarricense, 25, 53-60.

Burbano, E. A. (1990). "Efecto de la escarificación química en la calidad de semilla de Centrosema spp. durante el almacenamiento”. Agronomía Mesoamericana, 1, 63-67.

Camino, J. (1998). Ensayos para mejorar la germinación del nance Byrsonima spp. Tesis de ingeniero agrónomo. Tegucigalpa: Escuela Agrícola Panamericana, El Zamorano.

Castellano, G., et al. (2006). "Efecto de tratamientos poscosecha sobre las características físico-químicas de las frutas de guayaba (Psidium guajava L.)”. Congreso Nacional de Fruticultura. Barquisimeto.

Chaves, A., et al. (1999). "Conservación refrigerada de semillas de Araucaria angustifolia (Bert.) O. Kuntze”. Bosque, 20, 117-124. 
Daniel, W. W. (1995). Bioestadística: bases para el análisis de las ciencias de la salud. México: Limusa.

Flores-Cuevas, N. A. (2008). Estudio de la recuperación de un suelo contaminado con hidrocarburos por diferentes estrategias de biorremediación a nivel microcosmos. Tesis de Ingeniería. Universidad de Quintana Roo.

Guerrero, M. y J. Herrera (1994). "The germination of Sesbania emerus (Fabaceae): Effect of immersion in sulphuric acid”. Revista de Biología Tropical, 42, 461-466.

Hermosillo-González, Y., et al. (2008). "Métodos inductivos para maximizar la germinación de semilla de germoplasma nativo en vivero para sistemas silvopastoriles en Nayarit, México”. Zootecnia Tropical, 26, 355-358.

Hernández-Gil, R., F. Rada y R. Silva (2010). "Crecimiento y desarrollo en plantas juveniles de apate (Tabebuia rosea (Bertol.) A. DC.) sometidos a inundación”. Pittieria, 34, 113-126.

Hernández-Vargas, G., L. R. Sánchez-Velásquez y F. Aragón (2001). “Tratamientos pregerminativos en cuatro especies arbóreas de uso forrajero de la selva baja caducifolia de la sierra de Manantlán”. Foresta Veracruzana, 3, 9-15.

INEGI (2001). Sintesis de información geográfica del estado de Tabasco. Aguascalientes: Instituto Nacional de Estadística y Geografía.

Inzunza, J. C. (2005). “Clasificación de los climas de Köppen”. Ciencia Ahora, 15, 1-15. Martínez, H. H. (1986). "Resultados del comportamiento de especies forestales plantadas en líneas de enriquecimiento en Bajo Calima, San José del Guaviare y Tumaco, Colombia”. Corporación Nacional de Investigación y Fomento: Serie Técnica, 19, 20-33.

Meza-Sánchez, R., F. H. Ruiz-Espinosa y J. Navejas-Jiménez (2009). Guía para la producción de plantación con especies nativas. México: Secretaría de Agricultura, Ganadería, Desarrollo Rural, Pesca y Alimentación/Instituto Nacional de Investigaciones Forestales, Agrícolas y Pecuarias/Centro de Investigación Regional Noroeste Campo Experimental Todos Santos.

Niembro-Rocas, A. (1988). Semillas de árboles y arbustos útiles de México: ontogenia y estructura. México: Limusa.

Niembro-Rocas, A. y E. O. Ramírez-García (2006). "Evaluación de la cantidad y calidad de semillas de caoba (Swietenia macrophylla King) procedentes de una plantación en el estado de Campeche, México". Foresta Veracruzana, 8, 21-28. Ochoa-Gaona, S., et al. (2008). Manual de semillas de especies forestales de las mon- 
tañas de Tenosique, Tabasco. San Cristóbal de Las Casas: El Colegio de la Frontera Sur.

---------- (2009). Prospección de especies arbóreas nativas promisorias para la fitorremediación y su influencia en el incremento de la diversidad de especies en los municipios de Cárdenas y Huimanguillo, Tabasco. Informe final del proyecto R0251 ACU 177-09. El Colegio de la Frontera Sur/Secretaría de Recursos Naturales y Protección Ambiental. Tabasco, México.

Padma, V., B. M. Reddy y G. Satyanarayana (1995). "Breaking dormancy in certain Acacia spp. by pre-sowing seed treatments". Seed Research, 21, 26-30.

Pérez-Hernández, I., et al. (2011). "Germinación y supervivencia de seis especies nativas de un bosque tropical de Tabasco, México”. Madera y Bosques, 17, 71-91.

Perozo-Castro, R., et al. (2003). "Tiempo de remojo y profundidad de siembra en semillas del patrón níspero criollo (Manilkara zapota (Van Roye) (Jaca) Gill) Sapotaceae". Revista Facultad Agronómica, 20, 10-20.

Quiroz-Marchant, I., et al. (2009). Vivero forestal: producción de plantas nativas a raíz cubierta. Santiago de Chile: Centro Tecnológico de la Planta Forestal.

Salvador-Figueroa, M., M. de L. Adriano-Anaya y C. Becerra-Ortiz (2005). "Efecto del remojo en agua sobre la germinación de semillas de Papaya Var. Maradol”. Revista Chapingo Serie Horticultura, 11, 27-30.

Stearn, W. T. (1992). Botanical Latin: History, Grammar, Syntax, Terminology and Vocabulary. Londres: David and Charles Publishing.

Valle-Doménech, A. y S. Ochoa-Gaona (2008). "Tratamientos pregerminativos y germinación en invernadero de semillas de especies forestales nativas de las selvas húmedas de la sierra de Tenosique, Tabasco". En Primer Encuentro de Investigación Científica de Tabasco. Memorias en extenso [cD]. División Académica Multidisciplinaria de los Ríos, Universidad Juárez Autónoma de Tabasco, abril de 2008.

Vargas-Simón, G., R. Pire y K. de la O de la O (2009). "Efecto de la longitud radicular al momento del transplante sobre el crecimiento vegetativo de cuijinicuil (Inga jinicuil Schtdl, \& Cham. ex. Ge. Don) en invernadero". Agrociencia, 43 (6), 603-608.

Zamora-Cornelio, L. F., et al. (2010). "Germinación de semillas y claves para la identificación de plántulas de seis especies arbóreas nativas de humedales del suroeste de México”. Revista de Biología Tropical, 58, 717-732. 